\title{
STABLE THERMOMAGNETIC WAVES IN HARD SUPERCONDUCTORS
}

\author{
N.A.Taylanov \\ Institute of Applied Physics, Department of Theoretical Physics, National University of \\ Uzbekistan, Tashkent, Uzbekistan e-mail: taylanov@iaph.tkt.uz
}

\begin{abstract}
The problem of the stability of a nonlinear thermomagnetic wave with respect to small thermal and electromagnetic perturbations in hard superconductors was studied. It is shown that spatially bounded solutions may correspond only to the perturbations decaying with time, which implies stability of the nonlinear thermomagnetic wave.
\end{abstract}

Previously [1], it was demonstrated that, depending on the surface conditions, stationary nonlinear thermomagnetic waves of two types, $E$ and $H$, may exist in a superconductor. However, the problem of stability of the nonlinear waves with respect to small thermal and electromagnetic perturbations in superconductors is insufficiently studied. We will consider the stability of a nonlinear thermomagnetic wave with respect to small thermal and electromagnetic perturbations in a superconductor occurring in the critical state. It will be demonstrated that spatially bounded solutions may correspond only to the perturbations decaying with time. The evolution of the thermal $(T)$ and electromagnetic $(E$ and $H)$ perturbations in a superconductor is described by a nonlinear thermal conductivity equation $[2]$

$$
\nu(T) \frac{d T}{d t}=\Delta[\kappa(T) \Delta T]+\vec{j} \vec{E},
$$

in combination with the Maxwell equations

$$
\operatorname{rot} \vec{E}=-\frac{1}{c} \frac{d \vec{H}}{d t}, \quad \operatorname{rot} \vec{H}=\frac{4 \pi}{c} \vec{j}
$$

and the critical state equation

$$
\vec{j}=\vec{j}_{c}(T, \vec{H})+\vec{j}_{r}(\vec{E}) .
$$

where $\nu=\nu(T)$ and $\kappa=\kappa(T)$ are the heat capacity and thermal conductivity coefficients, respectively; $\vec{j}_{c}$ is the critical current density; and $\vec{j}_{r}$ is the resistive current density. Let us consider a plane semiinfinite sample $(x>0)$, exposed to an external magnetic field $\vec{H}=\left(0,0, H_{e}\right)$, increasing with the time at a constant rate $\frac{d \vec{H}}{d t}=0$. According to the Maxwell equation (2), the sample will feature a vortex electric field $\vec{E}=\left(0, E_{e}, 0\right)$ parallel to the current density vector: $\vec{j}: \vec{E} \| \vec{j}$.

In order to describe the $j_{c}(T, H)$ function, we will use the Bean-London equation of the critical state $\left(\frac{d j_{c}}{d H}=0\right)[3]$. According to this model, the $j_{c}(T)$ function can be presented as 
$j(T)=j_{c}\left(T_{0}\right)\left[1-\frac{T-T_{0}}{T_{c}-T_{0}}\right]:$ where $j_{0}=j_{c}\left(T_{0}\right)$ is the equilibrium current density, $T_{c}$ is the critical temperature, and $T_{0}$ is the cooler temperature. The characteristic form of $j_{r}(E)$ in the region of sufficiently strong electric fields $\left(E>E_{f}\right)$ can be approximated by a piecewise linear function $j_{r} \approx \sigma_{f} E$. In the region of small field strengths $E<E_{f}$ the $j_{r}(E)$ function exhibits a nonlinear character related to a thermoactivated flux creep [4]. For an automodel solution of the type $\xi=x-v t$ describing a wave propagating at a constant velocity $v$ along the $x$ axis, the system of Eqs.( 1 )-(3) acquires the following form:

$$
\begin{gathered}
-v\left[N(T)-N\left(T_{0}\right)\right]=\kappa \frac{d T}{d \xi}-\frac{c^{2}}{4 \pi v} E^{2}, \\
\frac{d E}{d \xi}=-\frac{4 \pi v}{c^{2}} j, \\
E=\frac{v}{c} H .
\end{gathered}
$$

where $N(T)=\int_{0}^{T} \nu(T) d T$.

Excluding variables $T$ and $H$ with the aid of expressions (4) and (6) and taking into account the boundary condition $E(z \rightarrow-\infty)=E_{e}$, we arrive at a differential equation describing the distribution $E(z)$

$$
\frac{d^{2} E}{d z^{2}}+\beta \tau \frac{d E}{d z}+\frac{4 \pi v^{2}}{c^{2} E_{\kappa}}\left[N(T)-N\left(T_{0}\right)\right]-\frac{E^{2}}{2 E_{\kappa}}=0
$$

Here $z=\frac{\xi}{L}$ and $\beta=\frac{v t_{\kappa}}{L}$ are dimensionless parameters, $L=\frac{c H_{e}}{4 \pi j_{0}}$ the magnetic field penetration depth into the superconductor, $\tau=\frac{D_{t}}{D_{m}}$ is the ratio of the coefficients of thermal $D_{t}=\frac{\kappa}{\nu}$ and magnetic $D_{m}=\frac{c^{2}}{4 \pi \sigma_{f}}$ diffusion, $t_{\kappa}=\frac{\nu L^{2}}{\kappa}$ is the thermal diffusion time, and $E_{\kappa}=\frac{\kappa}{a L^{2}}$ is a constant parameter.

In the approximation of a weakly heated superconductor $\left(T-T_{0}\right)<<T_{0}$, the heat capacity $\nu$ and thermal conductivity $\kappa$ coefficients are weakly dependent on the temperature profile. As is well known (see, e.g., [4]), the magnetic flux variations in a hard superconductor occur at a much greater rate as compared to those of the heat transfer, so that $\tau<<1$ or $D_{t}<<D_{m}$. In this approximation, we may neglect the terms related to dissipative effects in Eq. (7), after which a solution to this equation can be presented in the following form [5]

$$
E(z)=\frac{E_{1}}{2}\left[1-t h \frac{\beta \tau}{2}\left(z-z_{0}\right)\right] .
$$

Relationship (8) describes the profile of a thermomagnetic shock wave propagating into the superconductor. In order to study the stability of a nonlinear wave with respect to small perturbations, it is convenient to write a solution to Eqs. (1)-(3) in the following form: 


$$
T(z, t)=T(z)+\delta T(z, t) \exp \left[\frac{\lambda t}{t_{\kappa}}\right], E(z, t)=E(z)+\delta E(z, t) \exp \left[\frac{\lambda t}{t_{\kappa}}\right],
$$

where $T(z)$ and $E(z)$ are the stationary solutions and $\delta T, \delta E$ are small perturbations. Substituting expressions (9) into Eqs. (1)- (3), assuming $\delta T, \delta E<<T, E$ in the limit of $\tau<<1$ (this corresponds to a "fast" instability, $\lambda>>\frac{\nu v^{2}}{\kappa}[4]$ ), we obtain an equation for determining the eigenvalues of $\lambda[6]$

$$
\frac{d^{2} \epsilon}{d z^{2}}+\left[\frac{2}{c h^{2}}-\Lambda\right] \epsilon=0
$$

Using the substitution of variables of the type

$\xi=t h y, 1-\xi=2 s$, and $\epsilon(\xi)=\left(1-\xi^{2}\right)^{\frac{\Omega}{2}} \Phi(\xi)$, Eq. (10) can be reduced to a standart hypergeometric equation

$$
s(1-s) \frac{d^{2} \Phi}{d s^{2}}-[\Omega+1-2 s(\Omega+1)] \frac{d \Phi}{d s}-[\Omega(\Omega-1)-p(p-1)] \Phi=0
$$

Here, the even and odd integrals can be presented as

$$
\begin{gathered}
\Phi_{1}=F(\epsilon-1, \epsilon+2, \epsilon+1, s), \\
\Phi_{2}=s^{-\epsilon} F(-1,2,1-\epsilon, s),
\end{gathered}
$$

where $\mathrm{F}$ is the hypergeometric function [7]. The function must be finite at the singular point $s=1$. The $\epsilon$ values for which $\Phi_{1}$ is finite evidently correspond to a discrete spectrum: $\epsilon_{i}=0,1$ or $\lambda_{i}=-\tau^{-1}, 0$. The function $\Phi_{2}$ is finite only for $\epsilon=0$. Any solution in the form of a running wave is char- acterized by translational symmetry, which implies that a "perturbed" stationary profile $E(z)$ determined by Eq. (8) corresponds to the eigenvalue of the ground state $\lambda_{0}=0$. Differentiating Eq. (7) with respect to $z$, one may readily see that $\frac{d E_{0}}{d z}$ is an eigenfunction corresponding to $\lambda_{0}=0$. Indeed, the perturbation $\delta E=\frac{d E_{0}}{d z}$ essentially represents a small wave displacement. Thus, we may suggest that tha function $\frac{d E_{0}}{d z}$ exponentially tends to zero for $z \rightarrow+\infty$ and the corresponding eigenvalue is zero. Therefore, the problem cannot possess positive eigenvalues and $R e \lambda_{i}<0$. This result implies that the wave is stable with respect to relatively small thermal $\delta T$ and electromagnetic $\delta E$ fluctuations. The analysis of the second linearly-independent solution leads to the same conclusion.

\section{REFERENCE}

[1] I.L.Maksirnov,Yu.N.Mastakov, and N.A.Tay1anov, Fiz. Tverd. Tela (Leningrad) 28 (8), 2323 (1986) [Sov. Phys. Solid State 28, 1300 (1986)]. 
[2] N.A.Taylanov Superconduct. Science and Technology, 14, 326 (2001).

[3] C. P. Bean, Phys. Rev. Lett. 8 (6),250 (1962).

[4] R. G. Mints andA. L. Rakhrnanov, Instabilities in Superconductors (Nauka, Moscow, 1984).

[5] . V. I. Karprnan, Nonlinear Waves in Dispersive Media (Nauka, Moscow, 1973; Pergarnon, Oxford, 1975).

[6] L. D. Landau and E. M. Lifshitz, Quantum Mechanics: Nonrelativistic Theory (Fizrnatgiz, Moscow, 1963; Pergarnon, NewYork, 1977).

[7] D. S. Kuznetsov, Special Functions (Vysshaya Shkola, Moscow, 1965). 